\title{
Ganho de peso gestacional e macrossomia em uma coorte de mães e filhos
}

\author{
Gestational weight gain and macrosomia in a cohort of mothers and their children \\ Gilberto $\mathrm{Kac}^{1}$, Gustavo Velásquez-Meléndez ${ }^{2}$
}

\section{Resumo}

Objetivo: Identificar co-variávies potencialmente associadas à macrossomia, incluindo o ganho de peso gestacional excessivo.

Métodos: Foram investigados 230 pares de mães e filhos residentes no município do Rio de Janeiro, Brasil, utilizando-se desenho de coorte. A macrossomia, definida como valores de peso ao nascer $\geq 4.000 \mathrm{~g}$, constituiu-se na variável dependente. Os potenciais preditores da macrossomia foram avaliados através de análise estratificada e da técnica de regressão logística não-condicional, que estima a odds ratio (OR) e os respectivos intervalos de confiança (IC $95 \%$ ).

Resultados: A incidência de ganho de peso gestacional excessivo foi de $29,1 \%$, variando de $10 \%$ em mulheres com < 20 anos a $63,6 \%$ em mulheres que geraram filhos com macrossomia. A incidência geral de macrossomia foi de $4,8 \%$, sendo de $10,4 \%$ para os filhos de mulheres com ganho de peso gestacional excessivo e de $2,5 \%$ para os filhos de mulheres com ganho de peso gestacional normal. Mulheres com ganho de peso gestacional excessivo e $\geq 20$ anos apresentaram razão de chance não-ajustada 5,42 vezes maior de ter filhos com macrossomia (IC 95\%: 1,11-26,34). Apenas o ganho de peso gestacional excessivo (OR $=5,83$, IC $95 \%: 1,51-22,48)$ permaneceu associado à macrossomia no modelo final de regressão logística.

Conclusões: Considerando que o ganho de peso gestacional excessivo foi o único preditor associado à macrossomia, é importante que políticas de controle que considerem esse preditor sejam implementadas, evitando desfechos fetais desfavoráveis. Novos estudos, com um elenco maior de preditores e desfechos, devem ser realizados para a confirmação dos resultados.

J Pediatr (Rio J). 2005;81(1):47-53: Macrossomia fetal, peso ao nascer, ganho de peso, gravidez, obesidade, transição epidemiológica.

\begin{abstract}
Objective: To identify co-variables potentially associated to infant macrosomia, including excessive gestational weight gain.

Methods: A cohort was investigated consisting of 230 pairs of mothers and children, residents of the City of Rio de Janeiro. Fetal macrosomia, defined as a birth weight $\geq 4,000$ grams was treated as the dependent variable. Statistical analysis of the relation between macrosomia and co-variables involved stratified analysis and multivariate logistic regression, which estimates odds ratios (OR) and $95 \%$ confidence intervals (CI 95\%).
\end{abstract}

Results: The incidence of excessive gestational weight gain was $29.1 \%$, varying from $10 \%$ for women under 20 years to $63.6 \%$ for women who had given birth to children with macrosomia. Macrosomia incidence was $4.8 \%$ for the group as a whole, $10.4 \%$ for women with excessive gestational weight gain and $2.5 \%$ for women with normal gestational weight gain. Women with excessive gestational weight gain and $\geq 20$ years exhibited a chance of developing macrosomia that was 5.42 times greater (CI 95\%: $1.11-26.34$ ). Within the final multivariate logistic regression model, only excessive gestational weight gain $(O R=5.83$, CI 95\%: $1.51-22.48)$ remained associated to infant macrosomia.

Conclusions: Considering that excessive gestational weight gain was the only predictor related to macrosomia, it is important that preventive programs that take account of this predictor be implemented, avoiding undesirable fetal outcomes. Future studies should include a wider variety of macrosomia predictors and additional birth outcomes.

J Pediatr (Rio J). 2005;81(1):47-53: Fetal macrosomia, birth weight, weight gain, pregnancy, obesity, health transition.
1. Doutor. Departamento de Nutrição Social e Aplicada, Instituto de Nutrição, Universidade Federal do Rio de Janeiro (UFRJ), Rio de Janeiro, RJ.

2. Doutor. Departamento de Enfermagem Materno-Infantil e Saúde Pública, Escola de Enfermagem, Universidade Federal de Minas Gerais (UFMG), Belo Horizonte, MG.

Fontes de financiamento: Fundação Universitária José Bonifácio da Universidade Federal do Rio de Janeiro (FUJB/UFRJ) e Fundação de Amparo à Pesquisa do Estado do Rio de Janeiro (FAPERJ). Gilberto Kac e Gustavo Velásquez-Meléndez são bolsistas de produtividade do Conselho Nacional de Desenvolvimento Científico e Tecnológico (CNPq).

Artigo submetido em 30.06.04, aceito em 10.11.04.

Como citar este artigo: Kac G, Velásquez-Meléndez G. Ganho de peso gestacional e macrossomia em uma coorte de mães e filhos. J Pediatr (Rio J). 2005;81:47-53.

\section{Introdução}

Diversos estudos nacionais e internacionais foram realizados nas últimas décadas com o objetivo de investigar os determinantes, a magnitude e a evolução temporal do baixo peso ao nascer ${ }^{1-6}$. Em geral, esses estudos têm revelado que o ganho de peso gestacional insuficiente, a idade materna precoce, a baixa estatura materna, o consumo de álcool e tabaco, entre outros fatores, atuam como determinantes do baixo peso ao nascer.

É consenso na literatura que um ganho de peso adequado durante a gestação está associado a um desfecho fetal satisfatório ${ }^{7-9}$. Em geral, considera-se que o crescimento 
fetal normal é uma função positiva do ganho de peso gestacional, modificado pelo estado nutricional pré-gestacional $^{10}$. Na década passada, com o objetivo de otimizar o peso ao nascer, o Instituto de Medicina Americano (IOM) ${ }^{7}$ passou a recomendar ganhos de peso diferenciados segundo o estado nutricional materno, medido pelo índice de massa corporal (IMC) pré-gestacional. Desta forma, os ganhos deveriam ser maiores em mulheres com baixo IMC pré-gestacional ( $\left.<19,8 \mathrm{~kg} / \mathrm{m}^{2}\right)$, intermediários em mulheres com IMC pré-gestacional normal ( $>19,8 \mathrm{e} \leq 26,0 \mathrm{~kg} / \mathrm{m}^{2}$ ) e menores em mulheres com IMC pré-gestacional definidor de sobrepeso ( $>26,0$ e $\leq 29 \mathrm{~kg} / \mathrm{m}^{2}$ ) e de obesidade $\left(\text { IMC }>29,0 \mathrm{~kg} / \mathrm{m}^{2}\right)^{7}$.

Dados recentes sobre a magnitude do baixo peso ao nascer para a população brasileira revelaram que o problema continua apresentando relevância epidemiológica, estando, no momento, estável no que diz respeito à magnitu$\mathrm{de}^{4}$. Em contrapartida, estudos sobre o padrão de distribuição e sobre os determinantes da macrossomia ainda são pouco freqüentes na população brasileira.

Recentemente, alguns estudos internacionais ${ }^{11-12}$ e nacionais ${ }^{13-14}$ demonstraram uma estreita relação entre ganho de peso gestacional excessivo e macrossomia. A definição de macrossomia ainda constitui tema em debate, mas em geral ela tem sido definida como valores de peso ao nascer $\geq 4.000 \mathrm{~g}, \geq 4.500 \mathrm{~g}$ ou valores de peso ao nascer acima do percentil 90 da curva de referência para idade gestacional e sexo ${ }^{15}$. A ocorrência de macrossomia tem sido associada a um aumento no risco de cesáreas, trauma no parto e morbidade infantil, especialmente quando associada ao diabetes gestacional ${ }^{11}$. Entre os principais determinantes da macrossomia, destaca-se a idade materna avançada, a multiparidade, a obesidade pré-gestacional, além do ganho de peso gestacional excessivo ${ }^{16}$.

Dentro desse contexto, o objetivo do presente estudo consistiu em avaliar a associação entre macrossomia e ganho de peso gestacional excessivo, segundo as recomendações do IOM, a partir de dados de uma coorte de mães e filhos acompanhados durante 9 meses pós-parto.

\section{Métodos}

Quatrocentos e setenta e nove mulheres foram recrutadas e acompanhadas por 9 meses pós-parto em um estudo de coorte realizado no município do Rio de Janeiro, entre maio de 1999 e abril de 2001. Foram realizadas quatro ondas de seguimento, aos 15 dias, 2, 6 e 9 meses, aproximadamente, momentos nos quais dados antropométricos e todas as co-variáveis foram coletadas. As mulheres foram recrutadas em três locais distintos, a saber: na maternidade central da área, durante a rotina de pré-natal e durante a rotina de imunização do BCG; os dois últimos locais situavam-se no próprio centro municipal de saúde Marcolino Candau.

Os critérios de elegibilidade estabelecidos para entrada na coorte foram ter entre 15 e 45 anos, ter menos de 30 dias de pós-parto na data da primeira entrevista, estar livre de doenças crônicas, ter idade gestacional superior a 35 semanas ao nascimento, não apresentar gestação gemelar e residir na área programática do estudo.

O padrão de perdas foi avaliado levando em consideração a distribuição da taxa final de seguimento (número de mulheres com seguimento completo/número de mulheres que entraram na coorte), segundo diversas co-variáveis importantes. Diferenças nas taxas finais de seguimento foram avaliadas segundo o teste do qui-quadrado para proporções. Detalhes adicionais acerca do recrutamento, seleção de participantes, critérios de exclusão, coleta de dados antropométricos e padrão de perdas podem ser obtidos em publicações anteriores ${ }^{17-21}$.

A macrossomia (peso ao nascer $\geq 4.000$ gramas) foi definida como a variável dependente do presente estudo. 0 peso ao nascer foi informado pela mãe na primeira onda de seguimento, tendo sido feita a conferência com o valor registrado no cartão da criança sempre que o mesmo estava disponível. Testou-se a consistência da variável peso ao nascer com os valores de peso observados na primeira onda de seguimento, não tendo sido identificados valores inconsistentes, ou seja, os valores observados de peso ao nascer foram sempre inferiores aos observados na primeira onda de seguimento.

As seguintes co-variáveis foram incluídas na análise: sexo da criança (masculino, feminino), idade da mãe $(<20$, $\geq 20$ anos), cor de pele da mãe (branca, parda, negra), estado marital (casada, união, solteira), escolaridade $(<8$, $\geq 8$ anos), renda familiar total ( $<500, \geq 500$ reais), prática prévia de cesárea (não, sim), prática prévia de aborto (não, sim), paridade (um, $\geq 2$ filhos), idade da mãe ao primeiro parto ( $<17, \geq 17$ anos) e idade gestacional ao nascimento ( $<41, \geq 41$ semanas). A cor de pele da mãe foi obtida através de observação do entrevistador, e a idade gestacional calculada levando em consideração a data da última menstruação (DUM). Essa informação foi obtida na segunda onda de seguimento e tem sido sistematicamente usada para estimar a idade gestacional na ausência de dados baseados em ultra-sonografia ${ }^{22}$. O IMC [peso $(\mathrm{kg}) /$ estatura $\left(\mathrm{m}^{2}\right)$ ] pré-gestacional foi obtido na primeira onda de seguimento e foi calculado levando em consideração o peso prégestacional referido pela mãe e a estatura medida em estadiômetro Harpenden (Harpenden Inc.), segundo técnicas padronizadas ${ }^{23}$.

O ganho de peso gestacional foi a principal co-variável do estudo. Essa variável foi informada pela mãe na terceira onda de seguimento, aos 6 meses pós-parto, e analisada segundo categorias de ganho de peso recomendadas pelo IOM $^{7}$. O IOM recomenda ganhos diferenciados segundo o estado nutricional pré-gestacional, medido pelo IMC. Foram considerados como ganhos excessivos valores superiores a $18 \mathrm{~kg}$ para mulheres com IMC pré-gestacional $<19,8 \mathrm{~kg} / \mathrm{m}^{2}$, superiores a $16 \mathrm{~kg}$ para mulheres com IMC pré-gestacional entre 19,8 e $26 \mathrm{~kg} / \mathrm{m}^{2}$, superiores a $11,5 \mathrm{~kg}$ para mulheres com IMC pré-gestacional $>26$ e $\leq 29 \mathrm{~kg} / \mathrm{m}^{2}$, e $\geq 6 \mathrm{~kg}$ para mulheres com IMC pré-gestacional $>29 \mathrm{~kg} / \mathrm{m}^{2}$. Como dados de ganho de peso gestacional só foram obtidos na terceira onda de seguimento ( 6 meses pós-parto), ocorreram perdas de seguimento, e apenas 230 mulheres puderam ser incluídas na presente análise. 
A análise estatística envolveu inicialmente o teste de diferenças nas proporções de ganho de peso excessivo entre as co-variáveis descritas acima, usando o teste do qui-quadrado. No segundo momento, foram calculadas as incidências de macrossomia para o conjunto de crianças, segundo co-variáveis selecionadas, estratificando-se os resultados por ganho de peso gestacional excessivo ou não. Em seguida, foram calculados os riscos relativos (RR) estratificado, bruto e ajustado para ganho de peso gestacional, assim como os respectivos intervalos de confiança de 95\% (IC 95\%). Os valores de RR entre os estratos foram comparados usando o teste de Mantel \& Haenzel. A seleção das categorias de referência para as variáveis foi feita considerando-se a menor incidência de macrossomia. Por fim, realizou-se análise de regressão logística não-condicional com controle para variáveis de confusão, tendo como variável dependente a macrossomia. Variáveis que apresentaram significância estatística na análise bivariada foram incluídas no modelo final.

O projeto foi submetido e aprovado pela Comissão de Ética do Núcleo de Estudos de Saúde Coletiva (NESC) da Universidade Federal do Rio de Janeiro (UFRJ) e está de acordo com os princípios éticos de não-maleficência, beneficência, justiça e autonomia contidos na Resolução 196/96 do Conselho Nacional de Saúde ${ }^{24}$. Todas as participantes assinaram termo de consentimento, obtido de forma livre e espontânea após terem sido feitos todos os esclarecimentos necessários.

Todas as análises foram desenvolvidas no programa SPLUS 2000 (MathSoft). Valores de $p<0,05$ foram definidos como estatisticamente significativos.

\section{Resultados}

Análises comparativas revelaram que a incidência de macrossomia e as médias de peso ao nascer não diferiram entre as crianças que participaram desse estudo ( $\mathrm{n}=$ 230) e as consideradas perdas de seguimento ou aquelas para as quais as mães não possuíam dados de ganho de peso gestacional disponível $(n=175)$ (resultados não apresentados). A Tabela 1 apresenta dados sobre a taxa final de seguimento para diversas variáveis importantes do estudo. Observa-se que o padrão de perdas é aleatório, já que, para todas as variáveis testadas, o quiquadrado para proporções foi maior que 0,05.

Das 230 mulheres estudadas, 29,1\% apresentaram ganho de peso gestacional excessivo, $34,4 \%$ ganho de peso gestacional normal e $36,5 \%$ ganho de peso gestacional abaixo do recomendado pelo IOM. Os dados da Tabela 2 apresentam a distribuição de freqüência do ganho de peso

Tabela 1 - Distribuição de freqüência para variáveis selecionadas entre perdas, seguimento completo e taxa final de seguimento (Rio de Janeiro, 1991-2001)

\begin{tabular}{|c|c|c|c|c|c|}
\hline Variáveis & $\begin{array}{l}\text { No inicial de } \\
\text { observações }\end{array}$ & $\begin{array}{c}\text { Perdas de } \\
\text { seguimento } \\
\mathbf{n}\end{array}$ & $\begin{array}{l}\text { Seguimento } \\
\text { completo }\end{array}$ & $\begin{array}{c}\text { Taxa final de } \\
\text { seguimento } \\
\%\end{array}$ & $\mathbf{p}$ \\
\hline \multicolumn{6}{|c|}{ Faixa etária (anos) } \\
\hline $18-24$ & 196 & 95 & 101 & 51,5 & \\
\hline $25-29$ & 114 & 47 & 67 & 58,7 & \\
\hline $30-45$ & 95 & 33 & 62 & 65,3 & 0,0753 \\
\hline \multicolumn{6}{|l|}{ Estado marital } \\
\hline Solteira & 74 & 25 & 49 & 66,2 & \\
\hline União & 230 & 102 & 128 & 55,6 & \\
\hline Casada & 101 & 48 & 53 & 52,5 & 0,1680 \\
\hline \multicolumn{6}{|l|}{ Cor de pele } \\
\hline Branca & 154 & 59 & 95 & 61,7 & \\
\hline Parda & 179 & 79 & 100 & 55,9 & \\
\hline Negra & 72 & 37 & 35 & 48,6 & 0,1711 \\
\hline \multicolumn{6}{|c|}{ Sexo da criança } \\
\hline Masculino & 199 & 88 & 111 & 55,8 & \\
\hline Feminino & 206 & 87 & 119 & 57,8 & 0,6863 \\
\hline \multicolumn{6}{|c|}{ IMC pré-gestacional $\left(\mathrm{kg} / \mathrm{m}^{2}\right)$} \\
\hline$<19,8$ & 85 & 39 & 46 & 54,1 & \\
\hline $19,8-26,0$ & 249 & 103 & 146 & 58,6 & \\
\hline $26,1-29,0$ & 47 & 23 & 24 & 51,1 & \\
\hline$>29,0$ & 24 & 10 & 14 & 58,3 & 0,7424 \\
\hline \multicolumn{6}{|l|}{ Macrossomia } \\
\hline Sim & 20 & 9 & 11 & 55,0 & \\
\hline Não & 385 & 166 & 219 & 56,9 & 0,8683 \\
\hline
\end{tabular}

IMC = índice de massa corporal. 
gestacional excessivo segundo variáveis selecionadas. As maiores incidências de ganho de peso gestacional excessivo foram observadas entre mulheres casadas $(35,8 \%)$, com idade ao primeiro parto $<17$ anos $(36,6 \%)$, com idade gestacional $\geq 41$ semanas $(38,1 \%)$ e com peso ao nascer $\geq 4.000$ gramas $(63,6 \%)$. Não se observou diferença estatisticamente significativa na incidência de ganho de peso gestacional excessivo entre as categorias das diversas variáveis estudadas, sendo exceção o peso ao nascer $\geq 4.000$ gramas.

Tabela 2 - Incidência de ganho de peso gestacional excessivo, segundo variáveis selecionadas (Rio de Janeiro, 19992001)

\begin{tabular}{|c|c|c|c|c|}
\hline \multirow[t]{2}{*}{ Variáveis } & \multirow[b]{2}{*}{$\mathbf{n}$} & \multicolumn{2}{|c|}{$\begin{array}{c}\text { Ganho de peso } \\
\text { gestacional }\end{array}$} & \multirow[b]{2}{*}{$\mathbf{p}$} \\
\hline & & $\mathbf{n}$ & $\%$ & \\
\hline \multicolumn{5}{|c|}{ Sexo da criança } \\
\hline Masculino & 111 & 29 & 26,1 & \\
\hline Feminino & 119 & 38 & 31,9 & 0,4103 \\
\hline \multicolumn{5}{|c|}{ Idade da mãe (anos) } \\
\hline$<20$ & 20 & 2 & 10,0 & \\
\hline$\geq 20$ & 210 & 65 & 31,0 & 0,0867 \\
\hline \multicolumn{5}{|l|}{ Cor de pele } \\
\hline Branca & 95 & 33 & 34,7 & \\
\hline Parda & 100 & 23 & 23,0 & \\
\hline Negra & 35 & 11 & 31,4 & 0,1867 \\
\hline \multicolumn{5}{|c|}{ Estado marital } \\
\hline Casada & 53 & 19 & 35,8 & \\
\hline União & 128 & 37 & 28,9 & \\
\hline Solteira & 49 & 11 & 22,4 & 0,3293 \\
\hline \multicolumn{5}{|c|}{ Escolaridade (anos) } \\
\hline$<8$ & 102 & 30 & 29,4 & \\
\hline$\geq 8$ & 128 & 37 & 28,9 & 0,9503 \\
\hline \multicolumn{5}{|c|}{ Renda familiar total (reais) } \\
\hline$<500$ & 113 & 34 & 30,1 & \\
\hline$\geq 500$ & 117 & 33 & 28,2 & 0,8656 \\
\hline \multicolumn{5}{|c|}{ Prática prévia de cesárea } \\
\hline Não & 143 & 27 & 31,0 & \\
\hline Sim & 87 & 40 & 28,0 & 0,7292 \\
\hline \multicolumn{5}{|c|}{ Prática prévia de aborto } \\
\hline Não & 153 & 40 & 26,1 & \\
\hline Sim & 77 & 27 & 35,1 & 0,2107 \\
\hline \multicolumn{5}{|c|}{ Paridade (número de filhos) } \\
\hline 1 & 110 & 29 & 26,4 & \\
\hline$\geq 2$ & 120 & 38 & 31,7 & 0,4599 \\
\hline \multicolumn{5}{|c|}{ Idade ao primeiro parto (anos) } \\
\hline$<17$ & 41 & 15 & 36,6 & \\
\hline$\geq 17$ & 189 & 52 & 27,5 & 0,3323 \\
\hline \multicolumn{5}{|c|}{ Idade gestacional (semanas) } \\
\hline$<41$ & 188 & 51 & 27,1 & \\
\hline$\geq 41$ & 42 & 16 & 38,1 & 0,2200 \\
\hline \multicolumn{5}{|c|}{ Peso ao nascer $(\mathrm{g})$} \\
\hline$<4.000$ & 219 & 60 & 27,4 & \\
\hline$\geq 4.000$ & 11 & 7 & 63,6 & 0,0159 \\
\hline Total & 230 & 67 & 29,1 & \\
\hline
\end{tabular}

* Ganho de peso gestacional classificado segundo proposta do Instituto de Medicina Americano (IOM, 1990).
A Tabela 3 apresenta dados de incidência de macrossomia para o conjunto de crianças, estratificados por ganho gestacional excessivo e normal e por variáveis selecionadas. A incidência geral de macrossomia foi de $4,8 \%$, sendo de $10,4 \%$ para os filhos de mulheres com ganho de peso gestacional excessivo e de $2,5 \%$ para os filhos de mulheres com ganho de peso gestacional normal. As maiores incidências de macrossomia foram observadas entre filhos de mulheres com ganho de peso gestacional excessivo e com idade $\geq 20$ anos $(50,0 \%)$, com renda familiar total $\geq 500$ reais $(15,2 \%)$ e com idade gestacional ao parto $\geq 41$ semanas $(18,8 \%)$

Dados de RR para macrossomia (estratificado, bruto e ajustado) em relação ao ganho de peso gestacional podem ser observados na Tabela 4 . As chances de desenvolver uma criança com macrossomia foram 5,42 (IC 95\%: 1,11-26,34) vezes maiores em mulheres com ganho de peso gestacional excessivo e com 20 anos ou mais; no entanto, os valores de RR não diferiram entre estratos de ganho de peso gestacional. Os valores de RR não foram estatisticamente significativos para nenhuma das demais variáveis.

Resultados do modelo final de regressão logística revelaram que o ganho de peso gestacional excessivo foi o único preditor estatisticamente significativo, conferindo uma chance 5,83 (IC 95\%: 1,51-22,48) vezes maior de desenvolver macrossomia. A idade da mãe ( $R R=4,38$, IC 95\%: 0,76$25,28)$ foi mantida no modelo final com o objetivo de controlar confundimento residual (Tabela 5).

\section{Discussão}

Os resultados do presente estudo revelaram que mulheres com ganho de peso gestacional excessivo apresentaram 5,83 vezes (IC $95 \%: 1,51-22,48$ ) mais chances de dar à luz uma criança com macrossomia.

O ganho de peso gestacional excessivo tem sido descrito como um importante fator de risco para uma série de desfechos fetais e maternos desfavoráveis $7,8,20,21$. Observou-se, no presente estudo, que quase um terço das mães investigadas apresentaram ganho de peso gestacional excessivo. Esses valores são coincidentes com os reportados por Nucci et al. ${ }^{14}$ para uma amostra de mais de 3.000 mulheres estudadas em seis capitais brasileiras. A incidência de ganho de peso gestacional excessivo variou de $10 \%$ para mães com menos de 20 anos a $63,6 \%$ para aquelas que geraram filhos com macrossomia.

A incidência de macrossomia no presente estudo foi de apenas $4,8 \%$. Esse valor pode ser considerado baixo se comparado a estudos com populações nativas americanas, onde a incidência atinge valores altos, entre 16 e 31\% $25-27$, ou mesmo quando comparados à população americana em geral, que apresentava taxas da ordem de $10 \%{ }^{28}$. Comparações com países europeus, como a Alemanha $(10,1 \%)^{29}$ e a Dinamarca, ambas em $1999(20,0 \%)^{30}$, demonstram que os valores aqui observados ainda são baixos em relação a essas populações. No Brasil, ainda são escassos estudos sobre a magnitude ou mesmo sobre os determinantes da macrossomia. Resultados de um estudo com 2.275 pares de 
Tabela 3 - Incidência de macrossomia de acordo com ganho de peso gestacional, segundo variáveis selecionadas (Rio de Janeiro, 1999-2001)

\begin{tabular}{|c|c|c|c|c|c|c|}
\hline \multirow[t]{3}{*}{ Variáveis } & \multicolumn{6}{|c|}{ Ganho de peso gestacional * } \\
\hline & \multicolumn{2}{|c|}{ Excessivo } & \multicolumn{2}{|c|}{ Normal } & \multicolumn{2}{|c|}{ Total } \\
\hline & $\mathbf{n}$ & $\%$ & $\mathbf{n}$ & $\%$ & $\mathbf{n}$ & $\%$ \\
\hline \multicolumn{7}{|c|}{ Sexo da criança } \\
\hline Masculino & 3 & 10,3 & 4 & 4,9 & 7 & 6,3 \\
\hline Feminino & 4 & 10,5 & 0 & 0 & 4 & 3,4 \\
\hline \multicolumn{7}{|c|}{ Idade da mãe (anos) } \\
\hline$\geq 20$ & 1 & 50,0 & 1 & 5,6 & 2 & 10,0 \\
\hline$<20$ & 6 & 9,2 & 3 & 2,1 & 9 & 4,3 \\
\hline \multicolumn{7}{|l|}{ Cor de pele } \\
\hline Branca & 4 & 12,1 & 2 & 3,2 & 6 & 6,3 \\
\hline Parda & 3 & 13,0 & 2 & 2,6 & 5 & 5,0 \\
\hline Negra & 0 & 0 & 0 & 0 & 0 & \\
\hline \multicolumn{7}{|c|}{ Estado marital } \\
\hline Casada & 2 & 10,5 & 1 & 2,9 & 3 & 5,7 \\
\hline União & 5 & 13,5 & 2 & 2,2 & 7 & 5,5 \\
\hline Solteira & 0 & 0 & 1 & 2,6 & 1 & 2,0 \\
\hline \multicolumn{7}{|c|}{ Escolaridade (anos) } \\
\hline$\geq 8$ & 4 & 10,8 & 3 & 3,3 & 7 & 5,5 \\
\hline$<8$ & 3 & 10,0 & 1 & 1,4 & 4 & 3,9 \\
\hline \multicolumn{7}{|c|}{ Renda familiar total (reais) } \\
\hline$\geq 500$ & 5 & 15,2 & 1 & 1,2 & 6 & 5,1 \\
\hline$<500$ & 2 & 5,9 & 3 & 3,8 & 5 & 4,4 \\
\hline \multicolumn{7}{|c|}{ Prática prévia de cesárea } \\
\hline Sim & 4 & 14,8 & 2 & 3,3 & 6 & 6,9 \\
\hline Não & 3 & 7,5 & 2 & 1,9 & 5 & 3,5 \\
\hline \multicolumn{7}{|c|}{ Prática prévia de aborto } \\
\hline Sim & 3 & 11,1 & 1 & 2,0 & 4 & 5,2 \\
\hline Não & 4 & 10,0 & 3 & 2,7 & 7 & 4,6 \\
\hline \multicolumn{7}{|c|}{ Paridade (número de filhos) } \\
\hline 1 & 3 & 10,3 & 2 & 2,5 & 5 & 4,5 \\
\hline$\geq 2$ & 4 & 10,5 & 3 & 2,4 & 6 & 5,0 \\
\hline \multicolumn{7}{|c|}{ Idade ao primeiro parto (anos) } \\
\hline$<17$ & 1 & 6,7 & 1 & 3,8 & 2 & 4,9 \\
\hline$\geq 17$ & 3 & 11,5 & 6 & 2,2 & 9 & 4,8 \\
\hline \multicolumn{7}{|c|}{ Idade gestacional (semanas) } \\
\hline$\geq 41$ & 3 & 18,8 & 1 & 3,8 & 4 & 9,5 \\
\hline$<41$ & 4 & 7,8 & 3 & 2,2 & 7 & 3,7 \\
\hline Total & 7 & 10,4 & 4 & 2,5 & 11 & 4,8 \\
\hline
\end{tabular}

* Ganho de peso gestacional classificado segundo proposta do Instituto de Medicina Americano (IOM, 1990).

mães e filhos revelaram incidência de $5,3 \% 13$, enquanto que Nucci et al. ${ }^{31}$, apesar de não reportar valores de freqüência, observaram risco $61 \%$ maior de desenvolver macrossomia em mulheres com IMC entre 25,0 e 29,9kg/m².

Considerando o contexto da transição nutricional, é importante destacar que a incidência de macrossomia e suas tendências históricas devem ser consideradas novos e relevantes indicadores. Nesse sentido, é interessante observar que a incidência de macrossomia no presente estudo é baixa. Esses valores acabam por inserir o Brasil dentro de um contexto intermediário da transição nutricional, onde uma das manifestações avançadas certamente consiste na elevada freqüência de macrossomia 29.

Entre os diversos fatores determinantes da macrossomia, estudos internacionais têm destacado a multiparidade, o sobrepeso ou a obesidade pré-gestacional, a idade materna avançada, a idade gestacional prolongada, o ganho de peso gestacional excessivo e a ocorrência de diabetes gestacional como os preditores mais importantes $11,16,29$. Todos esses potenciais preditores foram investigados no 
Tabela 4 - Risco relativo (IC 95\%) para macrossomia, estratificado e ajustado, segundo ganho de peso gestacional, para variáveis selecionadas (Rio de Janeiro, 1999-2001)

\begin{tabular}{|c|c|c|c|c|}
\hline \multirow[b]{3}{*}{ Variáveis $^{\dagger}$} & \multicolumn{4}{|c|}{ Ganho de peso gestacional * } \\
\hline & Excessivo & Normal & Bruto & Ajustado \\
\hline & \multicolumn{4}{|c|}{ Risco relativo (IC 95\%) } \\
\hline Sexo da criança & $0,98(0,24-4,05)$ & $-\neq$ & $1,88(0,57-6,29)$ & $2,13(0,62-7,33)$ \\
\hline Idade da mãe (anos) & $5,42(1,11-26,34)$ & $2,69(0,29-24,47)$ & $2,33(0,52-9,71)$ & $3,64(0,89-14,85)$ \\
\hline Escolaridade (anos) & $1,08(0,26-4,46)$ & $2,37(0,25-22,3)$ & $1,39(0,42-4,69)$ & $1,41(0,43-4,60)$ \\
\hline Renda familiar total (reais) & $2,58(0,54-12,36)$ & $0,31(0,03-2,95)$ & $1,16(0,37-3,79)$ & $1,19(0,38-3,73)$ \\
\hline Prática prévia de cesárea & $1,98(0,48-8,13)$ & $1,72(0,25-11,87)$ & $1,97(0,61-6,18)$ & $1,88(0,60-5,88)$ \\
\hline Prática prévia de aborto & $1,11(0,27-4,57)$ & $0,75(0,08-7,07)$ & $1,14(0,34-3,76)$ & $0,98(0,30-3,24)$ \\
\hline Paridade (número de filhos) & $1,02(0,25-4,20)$ & $0,99(0,14-6,85)$ & $1,10(0,35-3,50)$ & $1,01(0,32-3,16)$ \\
\hline Idade ao primeiro parto (anos) & $1,73(0,23-13,28)$ & $0,57(0,06-5,26)$ & $0,98(0,22-4,15)$ & $1,13(0,26-4,88)$ \\
\hline Idade gestacional (semanas) & $2,39(0,60-9,58)$ & $1,76(0,19-16,2)$ & $2,56(0,80-8,48)$ & $2,18(0,67-7,06)$ \\
\hline
\end{tabular}

* Ganho de peso gestacional classificado segundo proposta do Instituto de Medicina Americano (IOM, 1990).

$\dagger$ Categorias de referência: sexo (feminino), idade da mãe $(<20$ anos), escolaridade $(<8$ anos), renda familiar total $(<500$ reais), prática prévia de cesárea (não), prática prévia de aborto (não), paridade (um filho), idade ao primeiro parto ( $<17$ anos), idade gestacional ( $<41$ semanas).

¥ Nenhum caso de macrossomia foi observado nesse estrato.

presente trabalho, sendo exceção o diabetes gestacional; no entanto, apenas o ganho de peso gestacional excessivo associou-se significativamente à macrossomia. A idade materna apenas apresentou risco significativo não-ajustado. Entre os demais preditores, não se observou efeito da paridade nem da idade gestacional avançada, embora a incidência de macrossomia para mulheres com $\geq 41$ semanas e ganho de peso gestacional excessivo tenha sido de $18,8 \%$, mais de três vezes superior à incidência média global, de $4,8 \%$.

Algumas limitações do presente estudo merecem ser discutidas. Em primeiro lugar, deve-se considerar a expressiva redução no número de observações, decorrente de perdas de seguimento, já que a informação sobre ganho de

Tabela 5 - Modelo final de regressão logística para macrossomia (Rio de Janeiro, 1999-2001)

\begin{tabular}{lccc}
\hline Variáveis & OR & IC 95\% & p \\
\hline $\begin{array}{l}\text { Ganho de peso } \\
\text { gestacional excessivo * }\end{array}$ & & & \\
$\quad$ Não & 1,00 & & \\
$\quad$ Sim & 5,83 & $1,51-22,48$ & 0,0104 \\
Idade da mãe (anos) & & & \\
$\quad<20$ & 1,00 & & \\
$\quad \geq 20$ & 4,38 & $0,76-25,28$ & 0,0982
\end{tabular}

* Ganho de peso gestacional classificado segundo proposta do Instituto de Medicina Americano (IOM, 1990). peso gestacional só foi obtida na terceira onda de seguimento, aos 6 meses pós-parto. Entretanto, análises prévias na mesma população ${ }^{20}$ e outras realizadas para o presente estudo revelaram a existência de um padrão de perdas aleatório, o que garante, assim, que as 230 mulheres e crianças analisadas aqui representam o conjunto inicial de 479 pares de mãe e filhos. Uma outra limitação se refere ao fato de o dado sobre o ganho de peso gestacional ter sido informado pela mãe, o que potencialmente pode gerar algum tipo de viés. Acreditamos, no entanto, que o uso dessa informação não invalida as conclusões do estudo, uma vez que os resultados que envolvem essa variável são bastante consistentes, ou seja, o valor da OR é alto $(5,83)$, além do mesmo estar acompanhado de alta significância estatística ( $p$ valor $=0,0104$ ). Outra justificativa se refere ao fato de que análises anteriores ${ }^{20}$ revelaram diferenças significativas entre as diversas categorias de ganho de peso gestacional para o desfecho obesidade materna. Ademais, dados disponíveis para 3.082 gestantes residentes em seis capitais brasileiras, entre 1991 e 1995, revelaram média de ganho de peso gestacional de $12,7 \mathrm{~kg}^{14}$, valor muito próximo ao observado no presente estudo $(12,9 \mathrm{~kg})$, o que aponta no sentido oposto ao da presença de viés. O reduzido número de casos de macrossomia $(n=11)$ não chega a ser uma limitação, apesar de potencialmente configurar problema de amostra. Entretanto, mesmo com esse reduzido número de casos, foi possível demonstrar o efeito do ganho de peso gestacional excessivo como fator potencialmente associado à macrossomia. 
Com o objetivo de aumentar a consistência e a relevância dos resultados aqui apresentados, análises similares foram realizadas tendo como variável dependente o alto comprimento ao nascer, definido como valores do índice comprimento para idade acima de 2 escores $z$. É interessante constatar que, contrariamente ao verificado para peso ao nascer, não foram observados resultados estatisticamente significativos para o alto comprimento ao nascer, motivo pelo qual não são apresentados resultados relativos a essa variável dependente.

Em síntese, observou-se, a partir dos resultados da presente investigação, que o ganho de peso gestacional excessivo segundo recomendações do IOM constituiu-se em um fator potencialmente associado à macrossomia. Embora os valores de incidência de macrossomia sejam ainda baixos, é fundamental que a magnitude, os determinantes e as tendências históricas desse agravo sejam monitorados de forma sistemática, visto que a macrossomia já constitui um importante problema de saúde pública em países que se encontram em um estágio mais avançado da transição nutricional.

\section{Referências}

1. McCormick MC. The contribution of low birth weight to infant mortality and childhood morbidity. N Engl J Med. 1985;312:82-90.

2. Kramer MS. Determinants of low birth weight: methodological assessment and meta-analysis. Bull World Health Organ. 1987;65:663-737.

3. Horta BL, Barros FC, Halpern R, Victora CG. Baixo peso ao nascer em duas coortes de base populacional no Sul do Brasil. Cad Saúde Pública. 1996;12 (Supl 1):27-31.

4. Monteiro CA, Benício MH, Ortiz LP. Tendência secular do peso ao nascer na cidade de São Paulo (1976-1998). Rev Saúde Pública. 2000;34 (Supl 6):26-40.

5. Gama SG, Szwarcwald CL, Leal MD, Theme Filha MM. The pregnancy during adolescence as a risk factor for low birth weight, Brazil. Rev Saúde Pública. 2001;35:74-80.

6. Ramakrishnan U. Nutrition and low birth weight: from research to practice. Am J Clin Nutr. 2004;79:17-21.

7. Institute of Medicine (IOM). Nutrition during pregnancy, weight gain and nutrient supplements. Report of the Subcommittee on Nutritional Status and Weight Gain during Pregnancy. Washington, DC: National Academy Press; 1990.

8. Abrams B, Selvin S, Gunderson EP. Pregnancy weight gain: still controversial. Am J Clin Nutr. 2000;71(Suppl):S1233-41.

9. Galtier-Dereure F, Boegner C, Bringer J. Obesity and pregnancy: complications and cost. Am J Clin Nutr. 2000;71(Suppl 5):S1242-8.

10. Butte NF, Ellis KJ, Wong WW, Hopkinson JM, Smith EO. Composition of gestational weight gain impacts maternal fat retention and infant birth weight. Am J Obstet Gynecol. 2003; 189:1423-32.

11. Rodrigues S, Robinson EJ, Kramer MS, Gray-Donald K. High rates of infant macrosomia: a comparison of a Canadian native and a non-native population. J Nutr. 2000;130:806-12.

12. Jolly MC, Sebire NJ, Harris JP, Regan L, Robinson S. Risk factors for macrosomia and its clinical consequences: a study of 350,311 pregnancies. Eur J Obstet Gynecol Reprod Biol. 2003;111:9-14.
13. Lizo CL, Azevedo-Lizo Z, Aronson E, Segre EA. Relação entre ganho de peso materno e peso do récem nascido. J Pediatr (Rio J). $1998 ; 74: 114-8$.

14. Nucci LB, Duncan BB, Mengue SS, Branchtein L, Schmidt MI, Fleck ET. Assessment of weight gain during pregnancy in general prenatal care services in Brazil. Cad Saúde Pública. 2001a;17:1367-74.

15. Berard J, Dufour P, Vinatier D, Subtil D, Vanderstichele $S$, Monnier JC, et al. Macrosomia: risk factors and outcome. A study of the outcome concerning 100 cases $>4500 \mathrm{~g}$. Eur J Obstet Gynecol Reprod Biol. 1998;77:51-9.

16. ACOG. Technical Bulletin number 159. Fetal macrosomia. Int J Gynecol Obstet. 1992;39:341-5.

17. Kac G. Fatores determinantes da retenção de peso pós-parto em uma coorte de mulheres com nove meses de seguimento [tese]. São Paulo: Faculdade de Saúde Pública, USP; 2002.

18. Kac G, Benício MH, Valente JG, Velasquez-Melendez G. Postpartum weight retention among women in Rio de Janeiro: a follow-up study. Cad Saúde Pública. 2003;19(Supl 1):S149-61.

19. Kac G, Benício MH, Velásquez-Meléndez G, Valente JG, Struchiner CJ. Breastfeeding and postpartum weight retention in a cohort of Brazilian women. Am J Clin Nutr. 2004;79:487-93.

20. Kac G, Benício MH, Velásquez-Meléndez G, Valente JG, Struchiner CJ. Gestational weight gain and prepregnancy weight influence postpartum weight retention in a cohort of Brazilian women. J Nutr. 2004;134:661-6.

21. Kac G, Benício MH, Velásquez-Meléndez G, Valente JG. Nine months postpartum weight retention predictors for Brazilian women. Public Health Nutr. 2004;7:621-8.

22. Ministério da Saúde (MS). Assistência Pré-natal. Manual Técnico. Brasília: Ministério da Saúde, 2000.

23. Lohman TG, Roche AF, Martorell R. Anthropometric Standardization Reference Manual. Illinois: Human Kinetics Books; 1988.

24. Conselho Nacional de Saúde (CSN). Resolução no 196/96 sobre pesquisa envolvendo serem humanos. Bioética. 1996;4:415-25.

25. Armstrong IE, Robinson EJ, Gray-Donald K. Prevalence of low and high birthweight among the James Bay Cree of northern Quebec. Can J Public Health. 1998;89:419-20.

26. Caulfield LE, Harris SB, Whalen EA, Stugamori ME. Maternal nutritional status, diabetes and risk of macrosomia among native Canadian women. Early Human Dev. 1998;50:293-303.

27. Pettit DJ, Bennet PH, William C, Kwnoler H, Baird R, Aleck KA. Gestational diabetes mellitus and impaired glucose tolerance during pregnancy. Long-term effect on obesity and glucose tolerance in the offspring. Diabetes. 1985;34:119-22.

28. Boyd ME, Usher RH, McLean FH. Fetal macrosomia. Prediction, risks, proposed management. Obstet Gynecol. 1983;61:715-22.

29. Bergman RL, Richter R, Bergman KE, Plagemann A, Brauer $M$, Dudenhausen JW. Secular trends in neonatal macrosomia in Berlin. Influences of potential determinants. Paediatr Perinat Epidemiol. 2003;17:244-9.

30. Orskou J, Kesmodel U, Heriksen TB, Secher NJ. An increasing proportion of infants weigh more than 4000 grams at birth. Acta Obstet Gynecol Scand. 2001;809:931-6.

31. Nucci LB, Schmidt MI, Duncan BB, Fuchs SC, Fleck ET, Santos Britto MM. Nutritional status of pregnant women: prevalence and associated pregnancy outcomes. Rev Saúde Pública. 2001;35:502-7.

Correspondência:

Gilberto Kac

Universidade Federal do Rio de Janeiro - Instituto de Nutrição

Departamento de Nutrição Social e Aplicada

Avenida Brigadeiro Trompowsky s/no - Bloco J - $2^{\circ}$ andar

CEP 21941-590 - Rio de Janeiro, RJ

Fone: (21) 2562.6595 - Fax: (21) 2562.6695

E-mail: gkac@gbl.com.br 